\title{
Electrical Potential Difference during Laser Welding
}

\author{
${ }^{1}$ H. Zohm, ${ }^{2,3}$ G. Ambrosy and ${ }^{1}$ K. Lackner \\ ${ }^{I}$ Max-Planck-Institut für Plasmaphysik, Boltzmannstr. 2, D-85748 Garching, Germany \\ ${ }^{2}$ Institut für Strahlwerkzeuge, Universität Stuttgart, Pfaffenwaldring 43, D-70569 Stuttgart, Germany \\ ${ }^{3}$ Present adress: ALSTOM, Brown Boveri Str. 7, CH-5401 Baden, Switzerland \\ e-mail address of corresponding author: hartmut.zohm@ipp.mpg.de
}

\begin{abstract}
We present a new model for the generation of thermoelectric currents during laser welding, taking into account sheath effects at both contact points as well as the potential drop within the quasi-neutral plasma generated by the laser. We show that the model is in good agreement with experimentally measured electric potential difference between the hot and the cold part of the welded workpiece. In particular, all three elements of the model are needed to correctly reproduce the sign of the measured voltage difference. The mechanism proposed relies on the temperature dependence of the electron flux from the plasma to the workpiece and hence does not need thermoemission from the workpiece surface to explain the experimentally observed sign and magnitude of the potential drop.
\end{abstract}




\section{Introduction}

Plasma production during laser welding is a commonly observed phenomenon due to the high local power deposited by the laser [1]. The plasma above the weld pool can scatter, diffract or absorb laser light and hence, the understanding of the formation of this plasma has received attention [2]. Another process induced by the plasma is the generation of electrical voltages and currents since the plasma contains free electrical charges. Such currents have been observed between workpiece and gas nozzle [3] and it has been shown that applying a magnetic field can control the plasma. An explanation of the observed current between workpiece and nozzle has also been put forward on the basis of sheath theory [3], neglecting however the effect that sheaths will form at both the hot and the cold side. Experiments using applied magnetic fields when no current was flowing between workpiece and nozzle have clearly shown that the welding seam quality can be influenced, pointing towards the generation of closed current loops within the workpiece and weld pool [4]. Recently, the existence of such currents has been demonstrated experimentally and a first attempt to explain the observation has been made on the basis of a potential drop within the plasma [5], [6]. In this paper, we combine the theoretical considerations mentioned above to set up a model of the current generation taking into account the effect of the plasma as well as that of the sheath. We then analyse the prediction of this model with respect to the experimental observations and find good agreement. In particular, the sign of the current seems to be determined by the combination of both effects mentioned above, proving that it is important to take both of them into account. 


\section{Model for current generation during laser welding}

We consider an experiment where laser welding produces a plasma above the welding spot. There will be a temperature gradient in the plasma from the hot centre above the weld pool to the colder position where the plasma is in contact with the workpiece. This temperature gradient leads to a potential difference between these parts of the material, which will drive a current through the material. This potential difference has been measured using a shunt connecting two otherwise insulated parts of the workpiece during welding [5]. A voltage drop of more than 1 Volt could be detected, giving rise to a current directed from the colder part to the centre of the plasma. Fig. 1 shows the geometry.

In order to model the potential drop, we first use the Saha equation to determine the ionization ratio $I$ as function of the electron temperature $T_{e}$. We note here that the application of this relation does not necessarily require the species involved to have the same temperature (see e.g. [7] for an in-depth discussion) and use

$$
I\left(T_{e}\right)=\frac{n_{Z+1} n_{e}}{n_{Z}}=2 \frac{Q_{Z+1}}{Q_{Z}} \frac{\left(2 \pi m_{e} k T_{e}\right)^{3 / 2}}{h^{3}} e^{-W / k T_{e}}
$$

where $Q_{Z}$ is the partition function of the ionization state $Z, n_{e}, n_{Z}$ and $n_{Z+1}$ are the number densities of electrons, ions of charge state $Z$ and ions of charge state $Z+1$, respectively, and $W$ denotes the ionization energy from $Z$ to $Z+1$. Furthermore, $m_{e}$ is the electron mass and $h$ Planck's constant. To obtain absolute values of $n_{e}$, we will use the pressure balance

$$
n_{e} T_{e}+n_{0} T_{M}+n_{e} T_{M}=p_{0}
$$


where $p_{0}$ is the ambient pressure at the (idealized sharp) interface between metal vapour (temperature $T_{M}$ ) and plasma on the one side and air on the other side. Assuming singly ionized metal ions and quasineutrality $\left(n_{i}=n_{e}\right)$, Eqns (1) and (2) can be combined to yield

$$
n_{e}\left(T_{e}\right)=\frac{1}{2} \sqrt{\left(1+\frac{T_{e}}{T_{M}}\right)^{2} I\left(T_{e}\right)^{2}+\frac{p_{0}}{T_{M}} I\left(T_{e}\right)}-\frac{1}{2}\left(1+\frac{T_{e}}{T_{M}}\right) I\left(T_{e}\right)
$$

Next, we estimate the potential difference between two (electrically insulated) parts in contact with the metal plasma at electron temperatures $T_{e 1}$ and $T_{e 2}$. For this situation, Ohm's law including the temperature effects reads ([8], for a physical interpretation of the 'Thermoforce' $0.71 n_{e} \nabla T_{e}$ see [9])

$$
e n_{e} \nabla \Phi=0.71 n_{e} \nabla T_{e}+\nabla p_{e}=1.71 n_{e} \nabla T_{e}+T_{e} \nabla n_{e}
$$

where $\Phi$ is the electrostatic potential. Since the gradient appears everywhere, the whole integration can be expressed independently of the path as

$$
\frac{d(e \Phi)}{d T_{e}}=1.71+\frac{T_{e}}{n_{e}} \frac{d n_{e}}{d T_{e}}
$$

which can be integrated to yield

$$
e \Phi_{\text {plasma }}=1.71\left(T_{e 2}-T_{e 1}\right)+\int_{T_{e 1}}^{T_{e 2}} \frac{T e}{n e} \frac{d n_{e}}{d T_{e}} d T_{e}
$$


where $n_{e}\left(T_{e}\right)$ in the integral has to be inserted from Eqn. (3). Eqn. (6) can be integrated numerically to determine the contribution of the plasma to the potential difference. However, the total potential difference also has to take into account the sheath effect from the plasma wall contact. According to [10], this contribution is determined by

$$
e \Phi_{\text {sheath }}=0.5 T_{e} \ln \left[2 \pi \frac{m_{e}}{m_{i}}\left(1+\frac{T_{i}}{T_{e}}\right)\right]
$$

where $m_{i}$ is the ion mass and $e$ the (positively defined) elementary charge. Using (6) and (7), the total potential difference

$$
e \Phi_{\text {tot }}=e \Phi_{\text {plasma }}+e\left(\Phi_{\text {sheath }}\left(T_{e_{2}}\right)-\Phi_{\text {sheath }}\left(T_{e 1}\right)\right)
$$

can be evaluated numerically to compare with the experimental result from [5]. This will be done in the next section.

\section{Discussion}

We now model the experimental situation described in [5], where a workpiece made of Aluminum was used. We assume that the ambient pressure is given by atmospheric pressure, $p_{0}=10^{5} \mathrm{~Pa}$, and the metal vapour temperature is given by the evaporation temperature of $\mathrm{Al}$ $(2740 \mathrm{~K})$ so that $T_{M}=0.236 \mathrm{eV}$. In addition, we use $W=5.98 \mathrm{eV}$, where all Al properties are taken from [11]. The partition functions are taken from [12] and we use $Q_{Z+1}=1$, practically independent of $T_{e}$ for singly charged $\mathrm{Al}$ and $Q_{Z}=8.46$ at $T_{e}=1 \mathrm{eV}$. This value will decrease to 5.77 for the evaporation temperature of $0.23 \mathrm{eV}$, but as we will see below, the contribution of the degree of ionisation is important at the higher temperatures. Inserting the numbers, we 
can evaluate the function $n_{e}\left(T_{e}\right)$ according to Eqn. (3) for our conditions. Fig. 2 shows the degree of ionization $n_{e} /\left(n_{e}+n_{0}\right)$ and the electron density for these conditions.

We note that although the degree of ionization continuously increases with $T_{e}$, there is a maximum of the total electron density with $T_{e}$ due to the condition of fixed total pressure. This means that $d n_{e} / d T_{e}$ in Eqn. (6) becomes negative above a certain $T_{e}$ (about $0.9 \mathrm{eV}$ for the numbers given above) and the contribution of $\Phi_{\text {plasma }}$ to $\Phi_{t o t}$ becomes smaller with higher $T_{e}$. It should be noted that due to our idealized boundary condition that there the plasma region consists of $\mathrm{Al}$ vapour and plasma only, we tend to overestimate the electron density and in fact, typical measurements show somewhat lower values (see e.g. the table in [1]), but since it is mainly the temperature gradient that enters in the absolute value of (6), the value of the voltage is not much affected even if a lower value of $n_{e}$ is assumed taking into account a mixing with the surrounding air or gas.

We now evaluate the sheath effect from Eqn. (7). Using the numbers above, the $T_{e} / T_{i}$ contribution is small and the term is roughly linear (approximately $-4.5 T_{e}$ for $T_{i}=0$ and -4.2 $T_{e}$ for $\left.T_{e}=T_{i}\right)$. We can now evaluate the whole potential difference according to Eqn. (8). This is shown in Fig. 3, where the potential difference is plotted as function of the temperature difference $T_{e 2}-T_{e 1}$.

Since the sheath contribution is negative and the plasma contribution positive, the function exhibits a sign change, for our parameters around $T_{e 2}-T_{e 1}=1.2 \mathrm{eV}$, corresponding to an absolute value of $T_{e 2}$ of about $1.4 \mathrm{eV}$. The numerical value of the potential drop is in the range of 1-2 Volts, which corresponds well with the above mentioned similar value of the experimental voltage drop across the shunt. 
It remains to clarify which sign belongs to which experimental situation. For the plasma effect, the gradients of pressure and electric field point in the same direction. Hence, the hotter contact point ( $T_{e 2}$ in our notation) will be on higher potential. Short circuiting this by an external current path (as e.g. the shunt in the welding experiment) will lead to a (technical) current from the hotter to the colder point (note that inside the plasma, this current flows against the gradient of the electric potential, consistent with the fact that it is a generator). For the sheath effect, more electrons arrive at the hotter plate, so short circuiting would lead to an electron flow from hot to cold, which means a technical current from cold to hot. In the welding experiment, the current was measured to flow from the welding point through the Al material into the periphery. If one assumes that the plasma at the welding point is hotter than in the periphery, the conclusion is that the plasma effect dominates, and the difference between the contact points is less than $1.2 \mathrm{eV}(11600 \mathrm{~K})$. This seems very reasonable since on the one hand, central $\mathrm{T}_{\mathrm{e}}$ values of up to $1.5 \mathrm{eV}$ above the $\mathrm{Al}$ evaporation temperature have been measured [13], depending on the laser power and on the other hand, the plasma is at least partly heated by absorption of the laser power above the weld point, so the contact temperature at the weld point may be somewhat below the central $T_{e}$ value. In addition, the mixing of the Al vapour with the surrounding air will lead to a lower effective mass to be used in Eqn. (7), decreasing the sheath effect and opening an even larger temperature window for positive $\Phi_{t o t}$

\section{Conclusions}

We have developed a model description of the voltage drop occurring in laser weld experiments between the hot weld pool and the colder workpiece. This model takes into account both the potential drop along the plasma because of the finite temperature gradient as well as the difference in sheath potentials at the contact positions of different temperature. It 
can explain both magnitude and sign of the experimentally observed potential difference during laser welding. The sheath effect has been proposed as mechanism for the potential difference observed between the workpiece and the nozzle in [3], but neglecting the fact that two sheaths form, one at the hot end and one at the cold end. However, since the sheath effect alone cannot explain the sign of the current observed in [5], we conclude that both effects are important and should be taken into account when calculating currents induced by a plasma cloud forming above a locally heated metal surface. We also note that the mechanism described here does not rely on thermoemission of electrons from the surface of the workpiece since the potential drop is generated by electrons from the plasma charging up the surface. Thermoemission may well play a role in determining the magnitude of the current flowing in the workpiece, but is obviously not necessary to explain the experimentally observed potential difference. Finally, we note that this mechanism of current generation can be of importance also for other applications, such as the melting of metal layers in high power targets used in Nuclear Fusion research.

\section{References}

[1] Hügel, H. et al., Fundamentals of Laser Induced Processes, Landolt-Börnstein VIII/1C, Springer Verlag (2004) 3.

[2] Miller, R. et al., J. Appl. Phys. 61 (1990) 2045.

[3] Zhang, X. et al., J. Appl. Phys. 93 (2003) 8842.

[4] Kern, M. et al.,Welding Journal 79 (2000) 72.

[5] Ambrosy, G. et al., SPIE 6346 (2007) 63461Q.

[6] Ambrosy, G., Nutzung elektromagnetischer Volumenkräfte beim Laserstrahlschweissen, Forschungsberichte des IFSW, Herbert Utz Verlag, ISBN 978-3-8316-0925-3 (2009).

[7] Rat, V. et al., J. Phys. D 41 (2008) 183001. 
[8] Braginsikii, S.I., Review of Plasma Physics, (edited by M. Leontovich) Vol. 1 Consultants Bureau, New York (1965) 249-268.

[9] Kaufmann, M., Plasmaphysik und Fusionsforschung, Teubner Verlag, ISBN 3-51900349- X, 1st edition (2003) 119.

[10] Stangeby P.C., Phys. Fluids 27 (1984) 686.

[11] http://www.goodfellow.com/csp/active/STATIC/G/Aluminium.HTML

[12] http://physics.nist.gov/PhysRefData/ASD/levels_form.html

[13] Schellhorn, M. et al., SPIE 3092 (1997) 522. 


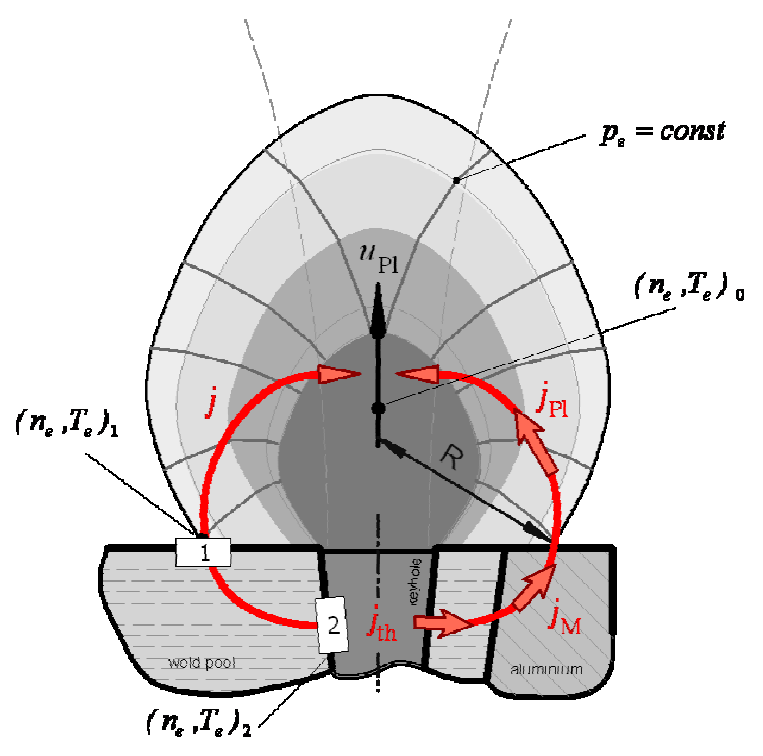

Fig. 1: Geometry of laser welding and experimentally determined direction of current flow. 

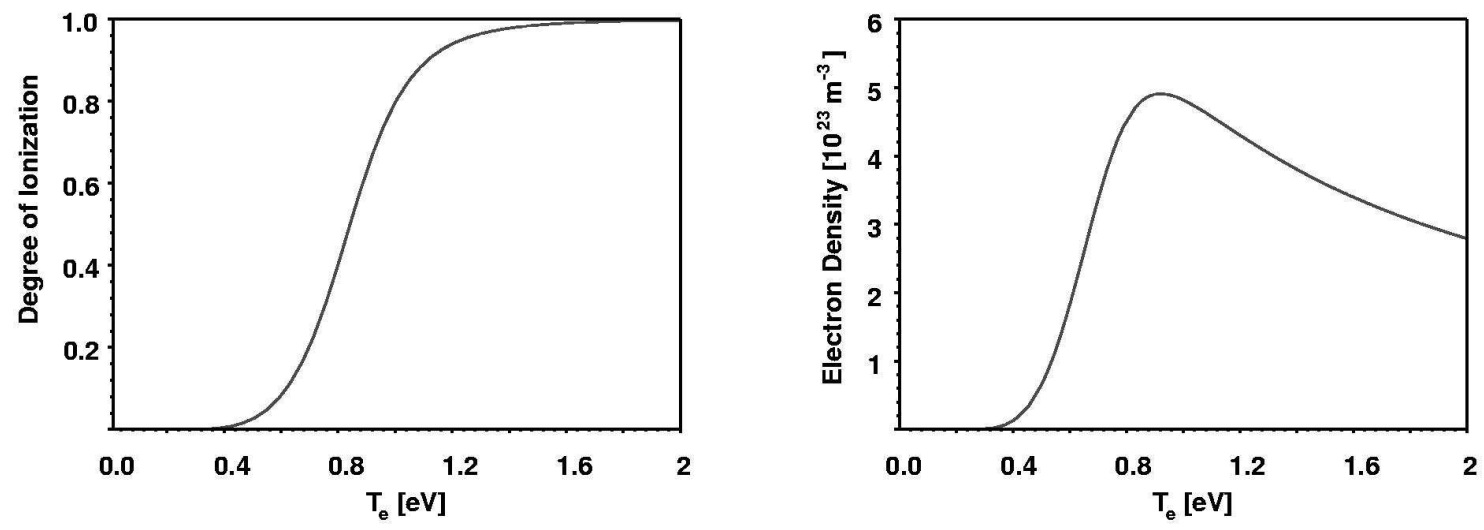

Fig. 2: Degree of ionization (left) and electron density (right) for Al laser welding using pressure equilibrium and the Saha equation. 


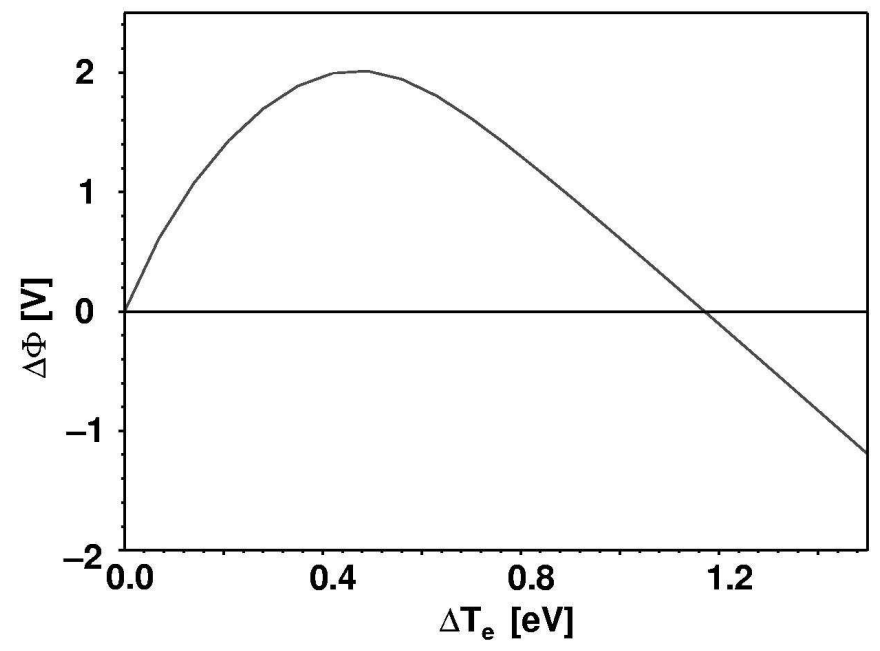

Fig. 3: Potential difference $\Delta \Phi$ as function of temperature difference $\Delta T_{e}=T_{e 2}-T_{e 1}$. 